\title{
Chiral symmetry breaking with a non-enhanced ghost sector
}

\section{Arlene C. Aguilar*}

Federal University of $A B C, C C N H$,

Rua Santa Adélia 166, CEP 09210-170, Santo André, Brazil.

E-mail: Arlene. Aguilardufabc.edu.br

We study chiral symmetry breaking using the quark gap equation supplemented with the infraredfinite gluon propagator and ghost dressing function obtained from large-volume lattice simulations. One of the most important ingredients of this analysis is the non-Abelian quark-gluon vertex, which displays a crucial dependence on the ghost dressing function and the quark-ghost scattering amplitude. The various theoretical ingredients necessary for this construction are reviewed in detail. As a result, we obtain a dynamical quark masses of the order of $300 \mathrm{MeV}$, which is used to compute phenomenological parameters such as the pion decay constant and the quark condensate.

The many faces of $Q C D$

November 2-5, 2010

Gent Belgium

${ }^{*}$ Speaker. 


\section{Introduction}

One of the most crucial features of QCD is the dynamical generation of a quark mass and the related phenomenon of chiral symmetry breaking (CSB). The realization of these characteristic QCD phenomena is intimately connected with the non-perturbative nature of the infrared sector of the theory; therefore, their study in the continuum is best studied within the inherently nonperturbative formalism based on the Schwinger-Dyson Equations (SDE) [1, 2].

It is well known that the non-linear integral equation governing the behavior of quark propagator (gap equation) displays a very rich structure. In particular, the existence or not of nontrivial solutions for this equation depends crucially on the strength of its kernel, which is largely determined by the non-perturbative behavior of the fully dressed gluon propagator and the quarkgluon vertex [1]. Actually, the quark-gluon vertex plays an absolutely essential role, introducing to the gap equation a dependence on the ghost dressing function [3] and the quark-ghost scattering amplitude [1]; as we will see, this dependence turns out to be numerically crucial for obtaining phenomenologically acceptable quark masses.

In this talk, we will show how it is possible to obtain physically relevant solutions for the quark dynamical mass using the infrared finite ingredients obtained from the lattice, i.e., a non-vanishing gluon propagator and a non-enhanced ghost sector. To do that, we build a truncation scheme for the quark SDE, supplemented with three non-perturbative ingredients: (i) the gluon propagator, (ii) the ghost dressing function obtained from large-volume lattice simulations $[4,5]$, and (iii) the "oneloop dressed" approximate version of the scalar form factor of the quark-ghost scattering kernel [1]. In addition, the results for the fermion masses in the adjoint representation are briefly discussed.

\section{The gap equation}

Let us start by defining some basic quantities that are important for the analysis of the CSB. The quantity that will be on the focus of our attention is the full quark propagator; in Minkowski space, its inverse has the general form given by

$$
S^{-1}(p)=\not p-m-\Sigma(p)=A\left(p^{2}\right) \not p-B\left(p^{2}\right),
$$

where $m$ is the bare current quark mass, and $\Sigma(p)$ the quark self-energy. Notice that the selfenergy can be decomposed in terms of a Dirac vector component, $A\left(p^{2}\right)$, and a scalar component, $B\left(p^{2}\right)$, which allow us to define the dynamical quark mass function as being the ratio $\mathscr{M}\left(p^{2}\right)=B\left(p^{2}\right) / A\left(p^{2}\right)$.

As we are interested in generating the quark mass exclusively through dynamical effects, we consider the case without explicit CSB, where the bare mass $m=0$. Then, it is easy to see that the quark propagator will develop a dynamical quark mass only if the scalar component, $B\left(p^{2}\right)$, is different from zero [6].

The diagrammatic representation of $S^{-1}(p)$ is shown in Fig.1; using the same convention of momenta flow as indicated in the figure, the gap equation can be written as

$$
S^{-1}(p)=\not p-C_{\mathrm{r}} g^{2} \int_{k} \Gamma_{\mu}^{[0]} S(k) \Gamma_{v}(-p, k, q) \Delta^{\mu v}(q),
$$


where $q \equiv p-k, \int_{k} \equiv \mu^{2 \varepsilon}(2 \pi)^{-d} \int d^{d} k$, with $d=4-\varepsilon$ the dimension of space-time. $C_{\mathrm{r}}$ is the Casimir eigenvalue of the given fermion representation, where we set $r=\mathrm{F}$ for the fundamental, and $r=\mathrm{A}$ for the adjoint. Specifically, for the gauge group $S U(3)$, we have $C_{\mathrm{A}}=3$ and $C_{\mathrm{F}}=4 / 3$.

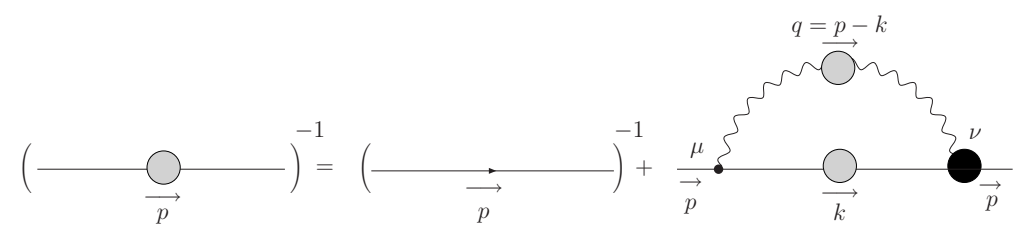

Figure 1: Diagrammatic representation of the quark SDE (gap equation).

According to Eq. (2.2), the quark propagator, $S(p)$, is dynamically determined in terms of an integral equation involving itself, the full gluon propagator, to be denoted by $\Delta^{\mu v}(q)$, the full fermion-gluon vertex $\Gamma_{v}(-p, k, q)$, and its tree level counterpart $\Gamma_{\mu}^{[0]}=\gamma_{\mu}$.

In the Landau gauge the full gluon propagator, $\Delta_{\mu v}(q)$, has the form

$$
\Delta^{\mu v}(q)=-i\left[g^{\mu v}-\frac{q^{\mu} q^{v}}{q^{2}}\right] \Delta\left(q^{2}\right)
$$

where the non-perturbative behavior of the scalar factor $\Delta\left(q^{2}\right)$ has been studied in great detail in the continuum $[7,8]$, and in the lattice simulations $[4,5]$.

\subsection{The full quark-gluon vertex}

In principle, the fully-dressed quark-gluon vertex, $\Gamma_{v}$, is determined from its own SDE, which contains a number of (unknown) multiparticle kernels, characteristic of the so-called "skeleton expansion". Dealing with such an equation is technically very difficult; therefore, the standard way to obtain information about this vertex is to use a gauge-technique Ansatz for it [10]. The general idea of this method is to express the longitudinal part of a vertex in terms of the various quantities appearing in the fundamental Ward identity or Slavnov-Taylor identity (STI) that it satisfies.

In the case of the quark-gluon vertex, the general Lorentz decomposition for the longitudinal part of $\Gamma_{v}$ involves four different form factors. All these form factors contain an explicit dependence to both the ghost dressing function and the "quark-ghost scattering kernel" which, in turn, also has a rich tensorial structure. Specifically, the vertex $\Gamma_{\mu}\left(p_{1}, p_{2}, p_{3}\right)$ satisfies the following STI [9]

$$
p_{3}^{\mu} \Gamma_{\mu}\left(p_{1}, p_{2}, p_{3}\right)=F\left(p_{3}\right)\left[S^{-1}\left(-p_{1}\right) H\left(p_{1}, p_{2}, p_{3}\right)-\bar{H}\left(p_{2}, p_{1}, p_{3}\right) S^{-1}\left(p_{2}\right)\right],
$$

where $H\left(p_{1}, p_{2}, p_{3}\right)$ is the quark-ghost scattering kernel represented in the Fig. 2, and $F\left(p_{3}\right)$ is the ghost dressing function which is related to the full ghost propagator by $D\left(p_{3}\right)=i F\left(p_{3}\right) / p_{3}^{2}$.

The kernel $H\left(p_{1}, p_{2}, p_{3}\right)$ and the "conjugate" $\bar{H}\left(p_{2}, p_{1}, p_{3}\right)$ have the following Lorentz decomposition [11] (note the change $p_{1} \leftrightarrow p_{2}$ in the arguments of the latter)

$$
\begin{aligned}
& H\left(p_{1}, p_{2}, p_{3}\right)=X_{0} \mathbb{I}+X_{1} \not p_{1}+X_{2} \not p_{2}+X_{3} \tilde{\sigma}_{\mu v} p_{1}^{\mu} p_{2}^{v}, \\
& \bar{H}\left(p_{2}, p_{1}, p_{3}\right)=\bar{X}_{0} \mathbb{I}-\bar{X}_{2} \not p_{1}-\bar{X}_{1} \not p_{2}+\bar{X}_{3} \tilde{\sigma}_{\mu v} p_{1}^{\mu} p_{2}^{v},
\end{aligned}
$$




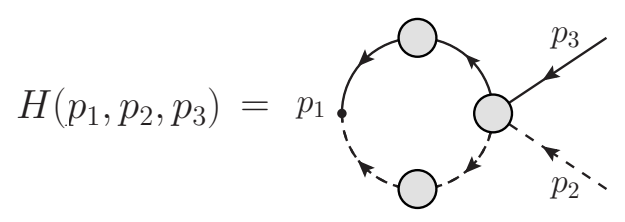

Figure 2: The fermion-ghost scattering kernel $H\left(p_{1}, p_{2}, p_{3}\right)$.

where the form factors $X_{i}$ are functions of the momenta, $X_{i}=X_{i}\left(p_{1}, p_{2}, p_{3}\right)$, and we use the notation $\bar{X}_{i}(p, r, q) \equiv X_{i}(r, p, q)$ and $\tilde{\sigma}_{\mu v} \equiv \frac{1}{2}\left[\gamma_{\mu}, \gamma_{v}\right]$.

The most general Lorentz decomposition for the longitudinal part of the vertex $\Gamma_{\mu}\left(p_{1}, p_{2}, p_{3}\right)$ can be written as [11]

$$
\Gamma_{\mu}\left(p_{1}, p_{2}, p_{3}\right)=L_{1} \gamma_{\mu}+L_{2}\left(\not p_{1}-\not p_{2}\right)\left(p_{1}-p_{2}\right)_{\mu}+L_{3}\left(p_{1}-p_{2}\right)_{\mu}+L_{4} \tilde{\sigma}_{\mu v}\left(p_{1}-p_{2}\right)^{v},
$$

where $L_{i}$ are the form factors, whose dependence on the momenta has been suppressed, in order to keep a compact notation, i.e., $L_{i}=L_{i}\left(p_{1}, p_{2}, p_{3}\right)$. Notice that the tree level expression for $\Gamma_{\mu}^{[0]}$ is recovered setting $L_{1}=1$ and $L_{2}=L_{3}=L_{4}=0$; then, $\Gamma_{\mu}^{[0]}=\gamma_{\mu}$.

Contracting Eq. (2.6) with $p_{3}^{\mu}$, we have

$$
p_{3}^{\mu} \Gamma_{\mu}\left(p_{1}, p_{2}, p_{3}\right)=\left(p_{2}^{2}-p_{1}^{2}\right) L_{3} \mathbb{I}+\left[\left(p_{2}^{2}-p_{1}^{2}\right) L_{2}-L_{1}\right] p_{1}-\left[\left(p_{2}^{2}-p_{1}^{2}\right) L_{2}+L_{1}\right] p_{2}-2 L_{4} \tilde{\sigma}_{\mu \nu} p_{1}^{\mu} p_{2}^{v} \text {. }
$$

In addition, substituting the standard decomposition of $S^{-1}(p)$, given in Eq. (2.1), and the expression of $H\left(p_{1}, p_{2}, p_{3}\right)$ given by Eq. (2.5) into Eq. (2.4), we find that the rhs of Eq. (2.7) can be also expressed in terms of the functions $A, B$ and $X_{i}$ 's. Then, it is relatively straightforward to demonstrate that the $L_{i}$ 's may be expressed as [1]

$$
\begin{aligned}
L_{1} & =\frac{F\left(p_{3}\right)}{2}\left\{A\left(p_{1}\right)\left[X_{0}+\left(p_{1}^{2}-p_{1} \cdot p_{2}\right) X_{3}\right]+A\left(p_{2}\right)\left[\bar{X}_{0}+\left(p_{2}^{2}-p_{1} \cdot p_{2}\right) \bar{X}_{3}\right]\right\} \\
& +\frac{F\left(p_{3}\right)}{2}\left\{B\left(p_{1}\right)\left(X_{1}+X_{2}\right)+B\left(p_{2}\right)\left(\bar{X}_{1}+\bar{X}_{2}\right)\right\} ; \\
L_{2} & =\frac{F\left(p_{3}\right)}{2\left(p_{2}^{2}-p_{1}^{2}\right)}\left\{A\left(p_{1}\right)\left[\left(p_{1}^{2}+p_{1} \cdot p_{2}\right) X_{3}-X_{0}\right]-A\left(p_{2}\right)\left[\left(p_{2}^{2}+p_{1} \cdot p_{2}\right) \bar{X}_{3}-\bar{X}_{0}\right]\right\} \\
& +\frac{F\left(p_{3}\right)}{2\left(p_{2}^{2}-p_{1}^{2}\right)}\left\{B\left(p_{1}\right)\left(X_{2}-X_{1}\right)+B\left(p_{2}\right)\left(\bar{X}_{1}-\bar{X}_{2}\right)\right\} ; \\
L_{3} & =-\frac{F\left(p_{3}\right)}{p_{2}^{2}-p_{1}^{2}}\left\{A\left(p_{1}\right)\left(p_{1}^{2} X_{1}+p_{1} \cdot p_{2} X_{2}\right)-A\left(p_{2}\right)\left(p_{2}^{2} \bar{X}_{1}+p_{1} \cdot p_{2} \bar{X}_{2}\right)+B\left(p_{1}\right) X_{0}-B\left(p_{2}\right) \bar{X}_{0}\right\} ; \\
L_{4} & =\frac{F\left(p_{3}\right)}{2}\left\{A\left(p_{1}\right) X_{2}-A\left(p_{2}\right) \bar{X}_{2}+B\left(p_{1}\right) X_{3}-B\left(p_{2}\right) \bar{X}_{3}\right\} .
\end{aligned}
$$

It is interesting to notice that setting in Eq. (2.8) $X_{0}=\bar{X}_{0}=1$ and $X_{i}=\bar{X}_{i}=0$, for $i \geq 1$, and $F\left(p_{3}\right)=1$, we obtain the following expressions

$$
\begin{array}{ll}
L_{1}=\frac{A\left(p_{1}\right)+A\left(p_{2}\right)}{2}, & L_{3}=\frac{B\left(p_{1}\right)-B\left(p_{2}\right)}{p_{1}^{2}-p_{2}^{2}}, \\
L_{2}=\frac{A\left(p_{1}\right)-A\left(p_{2}\right)}{2\left(p_{1}^{2}-p_{2}^{2}\right)}, & L_{4}=0
\end{array}
$$


which give rise to the so-called Ball-Chiu (BC) vertex [12],

$$
\begin{aligned}
\Gamma_{\mathrm{BC}}^{\mu}\left(p_{1}, p_{2}, p_{3}\right) & =\frac{A\left(p_{1}\right)+A\left(p_{2}\right)}{2} \gamma^{\mu} \\
& +\frac{\left(p_{1}-p_{2}\right)^{\mu}}{p_{1}^{2}-p_{2}^{2}}\left\{\left[A\left(p_{1}\right)-A\left(p_{2}\right)\right] \frac{\not p_{1}-\not p_{2}}{2}+\left[B\left(p_{1}\right)-B\left(p_{2}\right)\right]\right\},
\end{aligned}
$$

which is widely employed in the literature for studies of CSB [6].

In this work, we go beyond the Abelian version of the quark-gluon vertex expressed by Eq. (2.10), and we consider the case where only the scalar component of the quark-ghost scattering kernel is non-vanishing i.e. $X_{0} \neq 0$ while $X_{i}=\bar{X}_{i}=0$, for $i \leq 1$. In this limit, the expressions of Eq. (2.8) reduce to

$$
\begin{array}{ll}
L_{1}=F\left(p_{3}\right) X_{0}\left(p_{3}\right)\left[\frac{A\left(p_{1}\right)+A\left(p_{2}\right)}{2}\right], & L_{3}=F\left(p_{3}\right) X_{0}\left(p_{3}\right)\left[\frac{B\left(p_{1}\right)-B\left(p_{2}\right)}{p_{1}^{2}-p_{2}^{2}}\right], \\
L_{2}=F\left(p_{3}\right) X_{0}\left(p_{3}\right)\left[\frac{A\left(p_{1}\right)-A\left(p_{2}\right)}{2\left(p_{1}^{2}-p_{2}^{2}\right)}\right], & L_{4}=0 .
\end{array}
$$

According the above expression, the form factors $L_{i}$ 's display an explicit dependence on the product $F\left(p_{3}\right) X_{0}\left(p_{3}\right)$ which contains information about the infrared behavior of the ghost propagator [1]. Therefore, the information about the ghost sector enters into the gap equation Eq. (2.2) through the quark-gluon vertex of Eq. (2.6).

Since the transverse part of the quark-gluon vertex is not constrained by the STI given by Eq. (2.4), it is possible to add a transverse part to the vertex without violating the this important identity. The so-called Curtis and Pennington $(\mathrm{CP})$ vertex [13], to be denoted by $\Gamma_{\mathrm{CP}}^{\mu}$, modifies $\Gamma_{\mathrm{BC}}^{\mu}$ by an identically conserved term [6], such that

$$
\Gamma_{\mathrm{CP}}^{\mu}\left(p_{1}, p_{2}, p_{3}\right)=\Gamma_{\mathrm{BC}}^{\mu}\left(p_{1}, p_{2}, p_{3}\right)+\Gamma_{\mathrm{T}}^{\mu}\left(p_{1}, p_{2}, p_{3}\right)
$$

with

$$
\Gamma_{\mathrm{T}}^{\mu}\left(p_{1}, p_{2}, p_{3}\right)=\frac{\gamma^{\mu}\left(p_{2}^{2}-p_{1}^{2}\right)-\left(p_{1}-p_{2}\right)^{\mu}\left(\not p_{1}+\not p_{2}\right)}{2 d\left(p_{1}, p_{2}\right)}\left[A\left(p_{2}\right)-A\left(p_{1}\right)\right]
$$

where

$$
d\left(p_{1}, p_{2}\right)=\frac{1}{p_{1}^{2}+p_{2}^{2}}\left\{\left(p_{2}^{2}-p_{1}^{2}\right)^{2}+\left[\frac{B^{2}\left(p_{2}\right)}{A^{2}\left(p_{2}\right)}+\frac{B^{2}\left(p_{1}\right)}{A^{2}\left(p_{1}\right)}\right]^{2}\right\} .
$$

In analogy to what happens in the case of the $\mathrm{BC}$ vertex, the ghost effects due to $F\left(p_{3}\right)$ and the quark-ghost scattering kernel $X_{0}^{[1]}\left(p_{3}\right)$ will be incorporated into the CP vertex through a simple multiplication of its tensorial structure by the factor $F\left(p_{3}\right) X_{0}^{[1]}\left(p_{3}\right)$. Therefore, within our approximation, the "ghost-improved" versions of the vertex, to be denoted by $\bar{\Gamma}_{\mathrm{BC}}^{\mu}\left(p_{1}, p_{2}, p_{3}\right)$ and $\bar{\Gamma}_{\mathrm{CP}}^{\mu}\left(p_{1}, p_{2}, p_{3}\right)$, respectively, can be written as

$$
\begin{aligned}
& \bar{\Gamma}_{\mathrm{BC}}^{\mu}\left(p_{1}, p_{2}, p_{3}\right)=F\left(p_{3}\right) X_{0}^{[1]}\left(p_{3}\right) \Gamma_{\mathrm{BC}}^{\mu}\left(p_{1}, p_{2}, p_{3}\right), \\
& \bar{\Gamma}_{\mathrm{CP}}^{\mu}\left(p_{1}, p_{2}, p_{3}\right)=F\left(p_{3}\right) X_{0}^{[1]}\left(p_{3}\right) \Gamma_{\mathrm{CP}}^{\mu}\left(p_{1}, p_{2}, p_{3}\right) .
\end{aligned}
$$


Notice that the above equation in the limit of $X_{0}^{[1]}(q)=1$ reduces to the vertex employed in Ref. [3].

Substituting the vertex $\bar{\Gamma}_{\mathrm{BC}}^{\mu}\left(p_{1}, p_{2}, p_{3}\right)$ given by Eqs. (2.6) and (2.11) into the gap equation (2.2), and defining $p_{1}=-p, p_{2}=k$, and $p_{3}=q$, we arrive at the following coupled system for $A\left(p^{2}\right)$ and $B\left(p^{2}\right)$

$$
\begin{aligned}
& A\left(p^{2}\right)=1+C_{r} g^{2} Z_{c}^{-1} \int_{k} \frac{\mathscr{K}_{0}(p-k)}{A^{2}\left(k^{2}\right) k^{2}+B^{2}\left(k^{2}\right)} \mathscr{K}_{A}^{\mathrm{BC}}(k, p), \\
& B\left(p^{2}\right)=C_{r} g^{2} Z_{c}^{-1} \int_{k} \frac{\mathscr{K}_{0}(p-k)}{A^{2}\left(k^{2}\right) k^{2}+B^{2}\left(k^{2}\right)} \mathscr{K}_{B}^{\mathrm{BC}}(k, p),
\end{aligned}
$$

where the kernel $\mathscr{K}_{0}(q)$ corresponds to the part that is not altered by the tensorial structure of the quark-gluon vertex, namely

$$
\mathscr{K}_{0}(q)=\Delta(q) F(q) X_{0}^{[1]}(q),
$$

while the parts that are affected, $\mathscr{K}_{A}^{\mathrm{BC}}(k, p)$ and $\mathscr{K}_{B}^{\mathrm{BC}}(k, p)$, are given by [14]

$$
\begin{aligned}
\mathscr{K}_{A}^{\mathrm{BC}}(k, p)= & \frac{A\left(k^{2}\right)}{2 p^{2}}\left[A\left(k^{2}\right)+A\left(p^{2}\right)\right][3 p \cdot k-2 h(p, k)]-2 B\left(k^{2}\right) \Delta B\left(k^{2}, p^{2}\right) \frac{h(p, k)}{p^{2}} \\
& -A\left(k^{2}\right) \Delta A\left(k^{2}, p^{2}\right)\left[k^{2}-\frac{(k \cdot p)^{2}}{p^{2}}+2 \frac{k \cdot p}{p^{2}} h(p, k)\right] \\
\mathscr{K}_{B}^{\mathrm{BC}}(k, p)= & \frac{3}{2} B\left(k^{2}\right)\left[A\left(k^{2}\right)+A\left(p^{2}\right)\right]+2\left[B\left(k^{2}\right) \Delta A\left(k^{2}, p^{2}\right)-A\left(k^{2}\right) \Delta B\left(k^{2}, p^{2}\right)\right] h(p, k),
\end{aligned}
$$

where

$$
h(p, k) \equiv \frac{\left[k^{2} p^{2}-(k \cdot p)^{2}\right]}{q^{2}}
$$

and

$$
\Delta A\left(k^{2}, p^{2}\right) \equiv \frac{A\left(k^{2}\right)-A\left(p^{2}\right)}{k^{2}-p^{2}}, \quad \Delta B\left(k^{2}, p^{2}\right) \equiv \frac{B\left(k^{2}\right)-B\left(p^{2}\right)}{k^{2}-p^{2}} .
$$

Similarly, the effect of the vertex $\bar{\Gamma}_{\mathrm{CP}}^{\mu}\left(p_{1}, p_{2}, p_{3}\right)$ is to replace the kernels $\mathscr{K}_{A}^{\mathrm{BC}}(k, p)$ and $\mathscr{K}_{B}^{\mathrm{BC}}(k, p)$, appearing in Eq. (2.16) and (2.17), by $\mathscr{K}_{A}^{\mathrm{CP}}(k, p)$ and $\mathscr{K}_{B}^{\mathrm{CP}}(k, p)$, respectively, where

$$
\begin{aligned}
& \mathscr{K}_{A}^{C P}(k, p)=\mathscr{K}_{A}^{B C}(k, p)+\frac{3 k \cdot p}{2 p^{2}} A\left(k^{2}\right) \Delta A\left(k^{2}, p^{2}\right) \frac{\left(k^{2}-p^{2}\right)^{2}}{d(k, p)}, \\
& \mathscr{K}_{B}^{C P}(k, p)=\mathscr{K}_{B}^{B C}(k, p)+\frac{3}{2} B\left(k^{2}\right) \Delta A\left(k^{2}, p^{2}\right) \frac{\left(k^{2}-p^{2}\right)^{2}}{d(k, p)} .
\end{aligned}
$$

\section{The non-perturbative ingredients from the lattice}

As we have seen in Eq. 2.18, the gap equation given in Eqs. (2.16) and (2.17) depends on the nonperturbative form of the three basic Green's functions, namely $\Delta(q), F(q)$, and $X_{0}(q)$. Therefore, in order to proceed with the analysis, we use for $\Delta(q)$ and $F(q)$ the recent lattice data obtained by [4], and shown in Fig. 3 .

We clearly see that both lattice results for $\Delta(q)$ and $F(q)$ are infrared finite. This characteristic feature has been long associated with a purely non-perturbative effect, namely the dynamical generation of an effective (momentum-dependent) gluon mass [15, 2, 16], whose presence saturates 

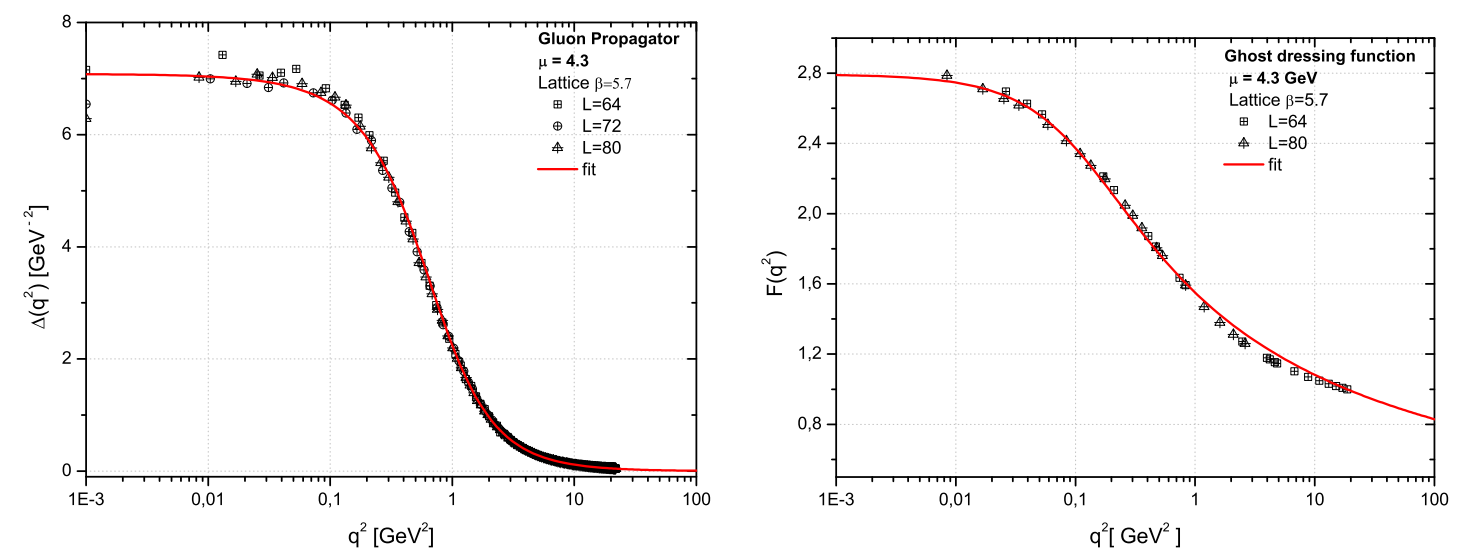

Figure 3: Lattice results for the gluon propagator, $\Delta(q)$, and ghost dressing, $F(q)$ renormalized at $\mu=4.3 \mathrm{GeV}$.

the gluon propagator in the IR. In addition, the appearance of the gluon mass is also responsible for the infrared finiteness of the ghost dressing function, $F\left(q^{2}\right)$ [7, 17], which is shown on the right panel of Fig. 3.

The only ingredient missing at this point is the form factor $X_{0}^{[1]}(q)$. Unfortunately, as far we know, there are no lattice data available for $X_{0}(q)$. We will therefore proceed with our analysis by deriving an estimate for $X_{0}$, based on its "one-loop dressed" approximation [1].

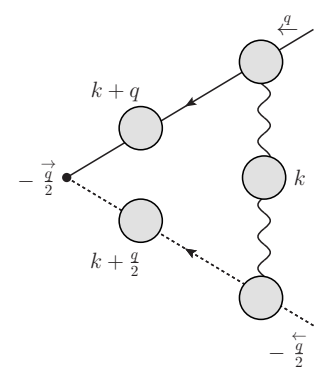

Figure 4: Diagrammatic representation of the quark-ghost scattering kernel, $H(-q / 2,-q / 2, q)$, at one-loop.

Specifically, we will consider the scalar contribution of the diagram represented in Fig. 4 and can be written as

$$
X_{0}^{[1]}(p, p, q)=1-i\left(\frac{1}{4}\right) \frac{C_{A} g^{2}}{2} \int_{k} \Delta^{\mu v}(k) D(k-p) G_{v} \operatorname{Tr}\left\{S(k+q) \Gamma_{\mu}\right\} .
$$

where the full gluon-ghost vertex is denoted by $G_{\mu}^{a b}=\delta^{a b} G_{\mu}$.

Using the tree-level expression for $G_{v}=(k-p)_{v}$, and the kinetic configuration where $p_{1}=p_{2} \equiv p$, and $p=-q / 2$, we notice that the above equation simplifies considerably (for more details see [1]), and we arrive at

$$
X_{0}^{[1]}(q)=1+\frac{1}{4} C_{A} g^{2} q^{2} \int_{k}\left[1-\frac{(k \cdot q)^{2}}{k^{2} q^{2}}\right] \Delta(k) F(k) \frac{F(k+q)}{(k+q)^{4}} .
$$


A non-perturbative estimate for $X_{0}^{[1]}(q)$ can be obtained by substituting into Eq. (3.2) the lattice data for $\Delta(q)$ and $F(q)$, given in Fig. 3. The numerical result for $X_{0}^{[1]}(q)$ is shown in the Fig. 5.

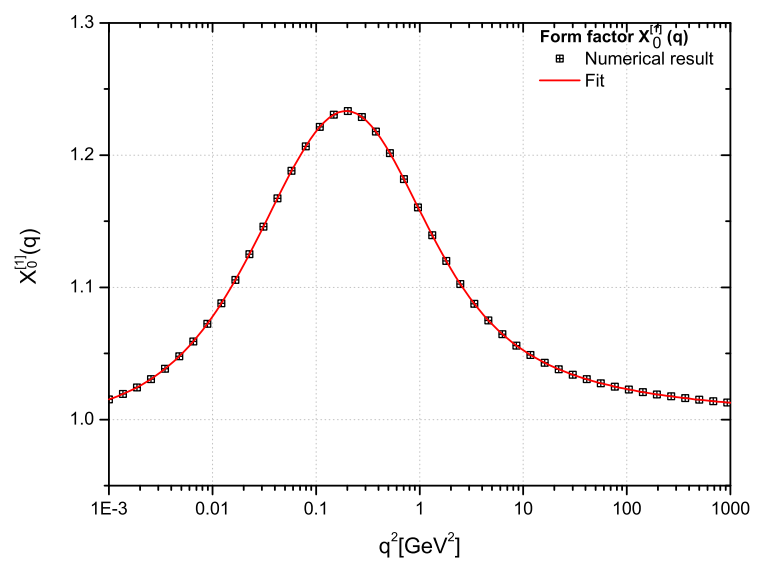

Figure 5: Numerical result for the form factor $X_{0}^{[1]}(q)$ of the quark-ghost scattering kernel given by Eq. (3.2) when $\alpha\left(\mu^{2}\right)=0.295$.

As we can see $X_{0}^{[1]}(q)$ shows a maximum located in the intermediate momentum region (around $450 \mathrm{MeV}$ ), while in the UV and IR regions $X_{0}^{[1]}(q) \rightarrow 1$. Although this peak is not very pronounced, it occurs in a region where the kernel of the gap equation is extremely sensitive. We have noticed that minor changes in the intermediate region result in considerable changes in the value of the dynamical mass generated as discussed in detail in Ref. [1]).

\section{Numerical results}

With all ingredients in hand, we are now in position to solve the system formed by Eqs. (2.16) and (2.17). Substituting $\Delta\left(q^{2}\right), F\left(q^{2}\right)$, and $X_{0}^{[1]}(q)$ into Eqs. (2.16) and (2.17), with the modification $Z_{c}^{-1} \mathscr{K}_{A, B}(k, p) \rightarrow \mathscr{K}_{A, B}(k, p) F\left(p^{2}\right)$, to enforce the correct renormalization group behavior of the dynamical mass (see discussion in [1]), we determine numerically the unknown functions $A\left(p^{2}\right)$ and $B\left(p^{2}\right)$ for the fundamental representation. The result for the quark wave function $A^{-1}\left(p^{2}\right)$ is shown in the left panel of Fig. 6, while the right panel shows the dynamical quark mass $\mathscr{M}\left(p^{2}\right)$.

Notice that Fig. 6 shows the result for both vertices $\bar{\Gamma}_{\mathrm{BC}}^{\mu}\left(p_{1}, p_{2}, p_{3}\right)$ (red dashed curves) and $\bar{\Gamma}_{\mathrm{CP}}^{\mu}\left(p_{1}, p_{2}, p_{3}\right)$ (black continuous line). One clearly sees that $\mathscr{M}\left(p^{2}\right)$ freezes out and acquires a finite value in the IR, $(\mathscr{M}(0)=294 \mathrm{MeV}$ in the case of the $\mathrm{BC}$ vertex, and $\mathscr{M}(0)=307 \mathrm{MeV}$ for the $\mathrm{CP}$ vertex). Moreover, in the UV it shows the expected perturbative behavior represented by the blue dashed curve.

Once the behavior of the dynamical quark mass has been determined, we have computed the pion decay constant and the quark condensate. For the pion decay constant we obtained $f_{\pi}=80.6 \mathrm{MeV}$ while for the condensate $\langle\bar{q} q\rangle\left(1 \mathrm{GeV}^{2}\right)=(217 \mathrm{MeV})^{3}$, which are in good agreement with phenomenological results [1]).

Next, we solve the system of Eqs. (2.16) and (2.17) in the adjoint representation i.e. $C_{\mathrm{r}}=C_{\mathrm{A}}=3$. When we switch from the fundamental to the adjoint representation, the overall effect in the gap 

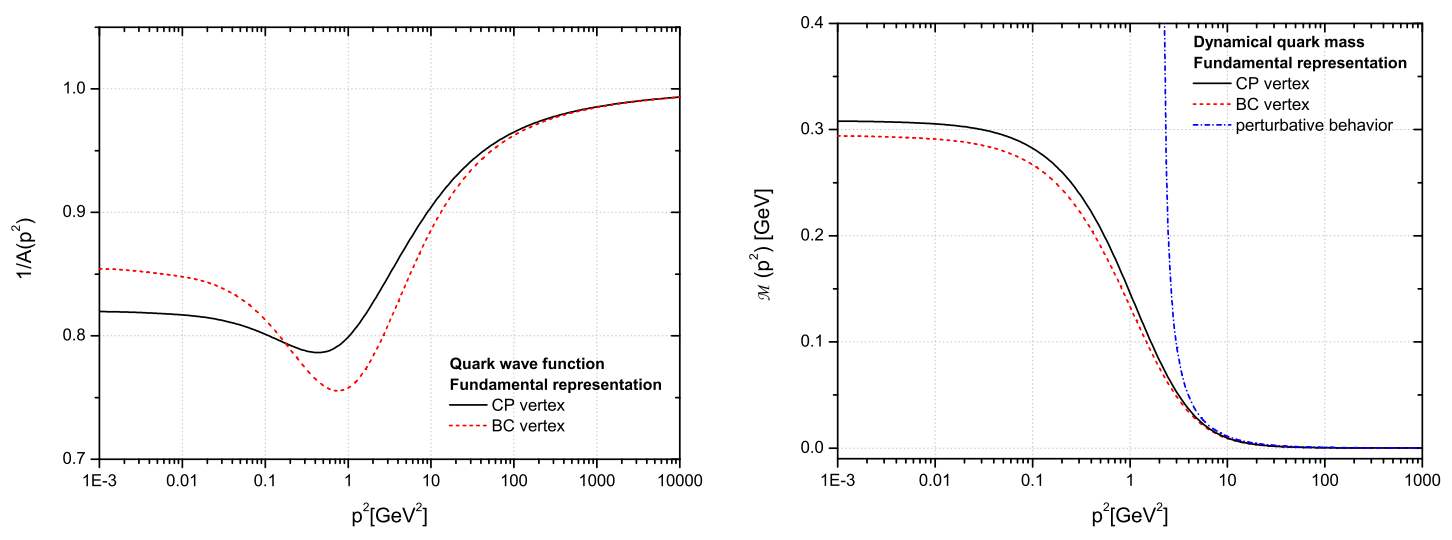

Figure 6: Left panel: The quark wave function $A^{-1}\left(p^{2}\right)$ in the fundamental representation for both vertices Right panel: The dynamical quark mass $\mathscr{M}\left(p^{2}\right)$.

equation is an enhancement factor of $9 / 4$ due to the difference in the corresponding Casimir eigenvalues.

The numerical results for the adjoint representation are shown in the Fig. 7. On the left panel, we compare the fermion wave functions, $A_{\text {adj }}^{-1}\left(p^{2}\right)$, when we use the modified BC (red dashed line) and $\mathrm{CP}$ vertices (black continuous line).

On the right panel, we show the fermion dynamical mass $\mathscr{M}_{\text {adj }}\left(p^{2}\right)$. We see that the infrared saturation of $\mathscr{M}_{\text {adj }}\left(p^{2}\right)$ occurs for higher values compared to the values of $\mathscr{M}\left(p^{2}\right)$ in the fundamental. More specifically, when the modified BC vertex is employed, one obtains $\mathscr{M}_{\text {adj }}(0)=750 \mathrm{MeV}$, while for the CP vertex $\mathscr{M}_{\text {adj }}(0)=962 \mathrm{MeV}$. Clearly, due to the nonlinear nature of the gap equation, the results found in the adjoint representation can not be reproduced from the fundamental solutions through a simple multiplication of the factor $9 / 4$. For example, the value for $\mathscr{M}_{\text {adj }}(0)$ are clearly higher than $9 / 4 \mathscr{M}(0)$.
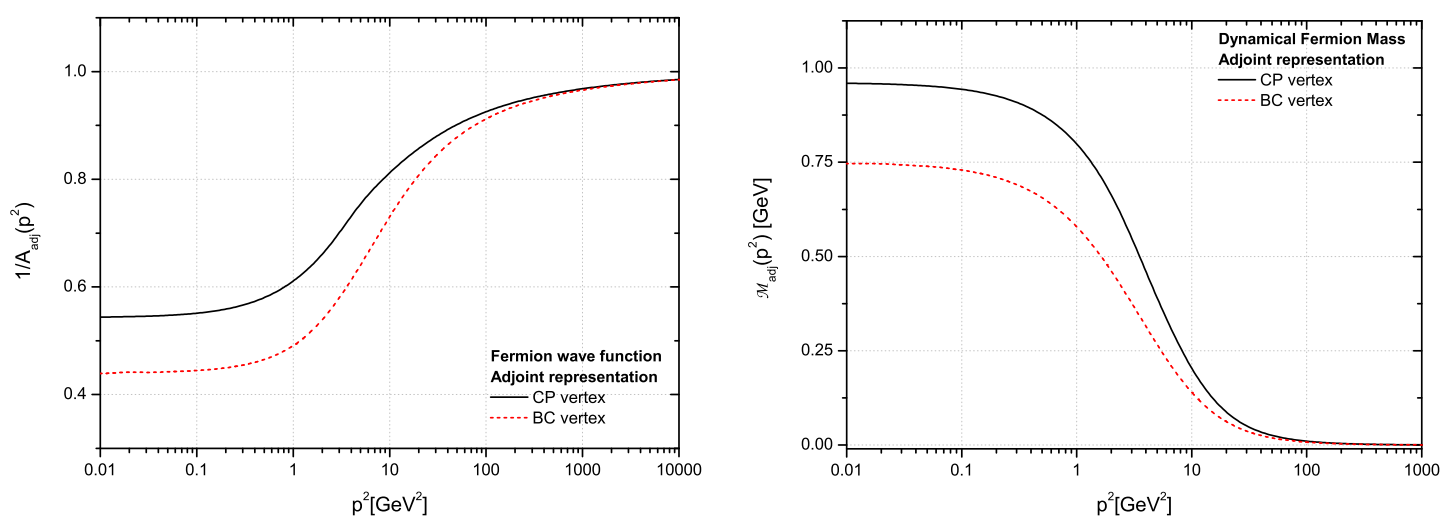

Figure 7: Left panel: The fermion wave function $A_{\text {adj }}^{-1}\left(p^{2}\right)$ in the adjoint representation. Right panel: The dynamical fermion mass $\mathscr{M}_{\text {adj }}\left(p^{2}\right)$. 


\section{Conclusions}

We have shown that the quark gap equation can give rise to phenomenologically compatible results for the dynamical quark mass provided that (i) a complete non-Abelian quark-gluon vertex (ghost-dressing function and quark-ghost scattering kernel) is introduced, and (ii) the recent lattice results for the gluon and ghost propagators are used.

It is important to emphasize that the incorporation of the appropriate ingredients in the way dictated by the underlying symmetry, as captured by the Slavnov-Taylor identity for the quarkgluon vertex, furnishes to the kernel (especially in the intermediate region of the momenta) the required support, thus obviating the need to resort to additional (artificially induced) enhancements, of questionable field-theoretic origin.

\section{Acknowledgments}

I would like to thank the organizers of the Many faces of QCD for the pleasant conference. This research is supported by the Brazilian Funding Agency CNPq under the grants 305850/2009-1 and 453118/2010-0.

\section{References}

[1] A. C. Aguilar and J. Papavassiliou, Phys. Rev. D 83, 014013 (2011).

[2] J. M. Cornwall and J. Papavassiliou, Phys. Rev. D 40, 3474 (1989).

[3] C. S. Fischer and R. Alkofer, Phys. Rev. D 67, 094020 (2003).

[4] I. L. Bogolubsky, E. M. Ilgenfritz, M. Muller-Preussker and A. Sternbeck, PoS LAT2007, 290 (2007).

[5] A. Cucchieri and T. Mendes, PoS LAT2007, 297 (2007); O. Oliveira and P. J. Silva, PoS QCD-TNT09, 033 (2009).

[6] C. D. Roberts and A. G. Williams, Prog. Part. Nucl. Phys. 33, 477 (1994).

[7] A. C. Aguilar and J. Papavassiliou, JHEP 0612, 012 (2006); A. C. Aguilar, D. Binosi and J. Papavassiliou, Phys. Rev. D 78, 025010 (2008); D. Binosi and J. Papavassiliou, Phys. Rept. 479, 1 (2009).

[8] D. Dudal, J. A. Gracey, S. P. Sorella, N. Vandersickel and H. Verschelde, Phys. Rev. D 78, 065047 (2008).

[9] W. J. Marciano and H. Pagels, Phys. Rept. 36, 137 (1978).

[10] A. Salam, Phys. Rev. 130, 1287 (1963); A. Salam and R. Delbourgo, Phys. Rev. 135, B1398 (1964).

[11] A. I. Davydychev, P. Osland and L. Saks, Phys. Rev. D 63, 014022 (2001).

[12] J. S. Ball and T. W. Chiu, Phys. Rev. D 22, 2542 (1980).

[13] D. C. Curtis and M. R. Pennington, Phys. Rev. D 42, 4165 (1990).

[14] F. T. Hawes, C. D. Roberts and A. G. Williams, Phys. Rev. D 49, 4683 (1994).

[15] J. M. Cornwall, Phys. Rev. D 26, 1453 (1982).

[16] A. C. Aguilar, A. Mihara and A. A. Natale, Phys. Rev. D 65, 054011 (2002)

[17] J. Rodriguez-Quintero, JHEP 1101, 105 (2011); arXiv:1012.0448 [hep-ph]. 